Uncovering the Information Needs in Complex Aerospace Systems

\author{
Iya Solodilova and Peter Johnson \\ Flightdeck Safety, Department of Computer Science, University of Bath \\ I.Solodilova@bath.ac.uk; P.Johnson@bath.ac.uk.
}

\begin{abstract}
A modern cockpit is the heart of a complex aerospace system. Representing complex information in a way that pilots can understand effectively remains a challenge. To understand how best to support pilots' information needs, we face the difficulty of having to study the complexity of the activity itself, the complexity of the environment, and the hidden nature of the information needs of the pilots. This paper shows how a "cue-recall debrief method" can be used as a powerful technique in investigating pilots' cognitive processes and activities. Moreover, it is claimed (Omodei, Wearing \& McLennan, 1997) that this technique has little effect on the complexity of the activity, operating environment and the pilot's experience. Using this method has uncovered distinct informationevolution stages, references, and strategies that pilots use when flying an automated aircraft.
\end{abstract}

\title{
Introduction
}

Research shows that pilots have difficulties understanding automated aircraft systems (Sarter \& Woods, 1994; 1995). Aviation Human Factors Experts involved in the design of advanced cockpits report that information content and the format of presentation on the interfaces gives little consideration to pilots' information needs (Newman, \& Greeley, 2001). The information content is based on an adhoc approach, influenced by previous designs and availability of latest technological trends.

The modern glass cockpit is a complex environment and the tasks required of modern pilots are similarly demanding. A modern pilot must be constantly monitoring the condition of the aircraft. This involves repeatedly switching focus between different instruments and displays, while efficiently guiding the aircraft to its destination and planning for what the aircraft will need to do in the future.

Various forms of analysis have been used to analyse this environment and the demands placed on the pilot. However, such analyses generally divide pilots' work reducing them into 'chunks' in a 'vertical' fashion.

However, we argue, that for time critical, dynamic and evolving environments accurate temporal flows cannot be easily preserved using these approaches, as 'chunks' cannot adequately depict the information flow that exists the modern cockpit.

Additionally, retrospective interviews and structured questionnaires are common techniques that are used to inform such approaches. The interview, for example, cannot capture the temporal aspects of the aerospace environment and pilots' activities. The questionnaire is restricted by the predetermined content of the questions, which cannot adapt to the answers of already answered questions by the pilot. These approaches, in most cases, look for confirmation of information that is already known to the researcher. They are poor on discovering from real-time observations and pilots' own interpretation of information that pilots use, for example the presentation form, frequency, quantity and quality of information.

Methods are required that trace the evolution of information from the beginning to end and that can be used to inform the future design of interfaces of complex systems.

\section{An Evolutionary Approach}

To address the last problem we have devised a three-step approach that aims to uncover pilots information needs in the complex domain of aerospace and informs interface design. The first step involves capturing real-time data, where a pilot wears a head-mounted camera whilst flying an uninterrupted flight from beginning to end. The second step, the cued-recall-debrief interview, takes place immediately after the flight where the pilot reviews captured video footage with the researcher. Both of these steps are based on the 'cued-recall-debrief' method (Omodei, Wearing \& McLennan, 1997), which we have tested and specifically modified for our approach during a preliminary study. The video footage captured from the pilot's point-of-view provides a powerful stimuli for “... evoking the recall of a wide range of cognitive and affective experiences with minimum distortion of the complexity and dynamics of these experiences" (Omodei, Wearing \& McLennan, 1997). This cuedrecall-debrief step reveals elements of pilots' thought processes and tracks pilots' needs for vital cues and information throughout the flight. The captured video footage is interpreted by the pilot and serves as a guide to a researcher in later analysis, which is the third step of the approach. 
An advantage of our three-step approach is that the empirical study and data analysis preserve the complexity of the environment and workflow, but do not influence it or interrupt it. In contrast to other observation studies where the researcher either interrupts the workflow, to ask questions about the thinking process of the operator, or asks the questions after the work has been completed, relying on the operator to recall the right moment and events that followed.

There are three main advantages to this approach (for more details, see Appendix). First, it acquires information without imposing a predetermined structure by a researcher. The structure and the content of information is guided by the events of the flight itself. Second, the probes for cueing pilot's comments and for identifying pilot's information requirements are provided through reliving the event by the pilot from his/her own-point-of-view. Third, the approach traces the evolution of information throughout the entire flight without interruption of any activities.

Steps One and Two of the Approach

Set up: Participants flew a full motion level five Hercules C130-J flight simulator on a regular flight from Sydney to Richmond. Each flight lasted between 15 to 20 minutes. Pilots flew one flight with full utilisation of automation and the second flight with minimum use of automation (see table 1).

\begin{tabular}{|c|c|c|c|c|c|}
\hline \multirow{2}{*}{\multicolumn{2}{|c|}{ Pilot }} & \multicolumn{2}{|c|}{ Automated } & \multicolumn{2}{|c|}{ Non-Automated } \\
\hline & & Flight & Debrief & Flight & Debrief \\
\hline \multirow{2}{*}{ Crew A } & 1 & $20 \mathrm{~min}$ & 1 hour $30 \mathrm{~min}$ & $20 \mathrm{~min}$ & 1 hour $30 \mathrm{~min}$ \\
\hline & 2 & $20 \min$ & 1 hour $30 \mathrm{~min}$ & $20 \mathrm{~min}$ & 1 hour $30 \mathrm{~min}$ \\
\hline \multirow{2}{*}{ Crew B } & 3 & $20 \mathrm{~min}$ & 1 hour $30 \mathrm{~min}$ & $20 \mathrm{~min}$ & 1 hour $30 \mathrm{~min}$ \\
\hline & 4 & $20 \mathrm{~min}$ & 1 hour $30 \mathrm{~min}$ & $20 \mathrm{~min}$ & 1 hour $30 \mathrm{~min}$ \\
\hline TOTAL & & 1 hour $20 \mathrm{~min}$ & 6 hours & 1 hour $20 \mathrm{~min}$ & 6 hours \\
\hline
\end{tabular}

Table 1 - Empirical Study set up

All flights for all participants were identical, and included three characteristics:

- A runway change aimed to increase pilots' workload. It allowed observation of how pilots dealt with new information and how they made changes to cockpit set up.

- Simulation of other traffic, on the radar display and on the radio to make the flight as realistic as possible.

- A cloud base between 1500 to 25000 feet to prevent pilots from seeing the ground at the top of a climb and to encourage pilots to rely on and use instruments throughout the flight. This also allowed pilots switching between instrument and visual operation during take-off and landing.

Rationale: The rationale behind observing an automated and non-automated flight was as follows:

1. Observation of an automated flight shows the problems pilots face and the workarounds pilots have invented to deal with recurrent problems with automation (Heymann, 2002). It also highlights where automation is most useful and is effortlessly used by pilots.

2. It well known that the Standard Operating Procedures that pilots use are devised, at least partly, to help pilots to overcome problems of poor automation design (also referred to as 'an indirect admittance of poor design', Demagalski, et al 2002).

3. Observing non-automated flight is less affected by automation-design-induced errors and shows pilots'-ways of dealing with information and 'pilot-like' operations, thus identifying activities that are based on deep-rooted 'flying experience'.

4. It is claimed that pilots use numerous strategies when collecting and using information in the automated and non-automated cockpit settings. Video footage captured both settings.

5. A non-automation fight operation focused on pilots using a more basic level of information available in the environment and the cockpit. In comparison, the fully automated flight focused on how pilots' obtained their necessary information with an 'abundance' of information available.

Rationale behind observation of the whole flight: We have observed the whole flight from 'power-up' to 'power down' to capture the following phenomena: (1) the aircraft environment is dynamic and time-critical, where current events are affected by past and present events and in turn affect subsequent events; (2) the information is also dynamic, constantly changing and dependent on evolution of all events. It is wrong to separate this information flow. Pilots deal with rising situations that are full of information that is dependent on the progress of the flight. The study of isolated stages of flight does not show the extent of how pilots build on and construct information, how information evolves and how having or not having a specific piece/s of information affects subsequent flight stages. 
Participants: The study involved observations of two crews (with two pilots in each crew) in a simulator. All participants were male military pilots. Pilots had on average $1600(\mathrm{SD}=663)$ total flying hours and had extensive experience (on average 825 ( $\mathrm{SD}=415$ ) flying hours) on the Electronic Flight Instrument System (i.e., an aircraft equipped with automation). All pilots had similar previous flying experience on both types of aircraft with and without use of automation (see table 2 below).

\begin{tabular}{|c|c|c|c|}
\hline \multirow{2}{*}{\multicolumn{2}{|c|}{ Pilot }} & \multicolumn{2}{|r|}{ Flying hours } \\
\hline & & Total & Electronic Flight Instrument Systems \\
\hline \multirow{2}{*}{ Crew A } & 1 & 1700 & 400 \\
\hline & 2 & 1100 & 800 \\
\hline \multirow{2}{*}{ Crew B } & 3 & 2500 & 1500 \\
\hline & 4 & 1100 & 600 \\
\hline \multicolumn{2}{|c|}{ Mean of flying hours } & 1600 & 825 \\
\hline
\end{tabular}

Table 2 - Flying experience

\section{Step Three: Evolutionary Data Analysis}

To avoid breaking down data as much as possible we have adapted an evolutionary data analysis technique that tracks links in data throughout the activity. The analysis can be thought of as a spiral, iterative progression through four stages (see Fig. 1: Evolution of the search): ( $1^{\text {st }}$ Stage) search for answers to posed questions; ( $2^{\text {nd }}$ Stage) search for commonalities and pattern; ( $3^{\text {rd }}$ Stage) identify properties transpired; and $\left(4^{\text {th }}\right.$ Stage $)$ search for additional data with transpired properties. Each stage of analysis allows refining the main posed question, hence allowing the uncovering of more detail for each posed question (see Fig.re 1).

The main posed questions at the beginning of the analysis are aimed at the direction of interest, without limiting the field of search too early. Each main posed question requires several spiralling iterations to refine the question until the question has been either explored in sufficient detail or it cannot be broken down any further into data that would inform the interface design or additional analysis is not required at this time. The questions become more specific and are refined further with every cycle through the stages (see Fig. 1).

Three main questions posed were:

Q1 - What information do pilots use to identify aircraft state?

Q2 - Does the information have structure and if so, what is that structure?

Q3 - Do pilots have strategies in assembling and using information to identify aircraft state?

Figure 1 shows how the first question (Q1 - Information) is refined through several iterations (see numbered arrows from question to question in Fig. 1). The next two main posed questions (Q2 Structure and Q3 - Strategy) would go through the same process as the first main posed question in figure 1 (see centre of the figure 'Q1 - Information'), with the only difference that at the centre of the figure there would be Q2 - Structure and Q3 - Strategy. Both of these questions would require their own iterations through four stages of analysis with surrounding questions aimed to answer the centre main poised question in required detail.

\section{Results}

The captured video footage (i.e., step one of the approach) and recorded cued-recall-debrief interview (i.e., step two of the approach) were transcribed to one document for each recorded flight for ease of analysis. This video data and the transcript were analysed using the evolutionary four-stage data analysis described above. We give several examples of data analysis for each main posed questions, followed by the summary of all results (see Fig. 2). The proceeding examples show a small proportion of the analysed data. It is timely at this stage to point out that all the analysis was done using real-time video footage and therefore the analysis is not removed from the original data.

\section{Q1 - 'What information do pilots use to identify aircraft state?}

The analysis began with identifying and collecting data to answer the first main posed question that explored the area of information (see centre in Fig. 1). During the first iteration through the data having the posed question in mind, we were looking for words that would identify 'information' that pilots use. The first words in the transcript that hinted to answer the question were 'referenced to' or 'referred to'. Around these words, we were likely to identify information that pilots used or 'referenced' in flight. Consider the following examples:

Pilot 04:42 M: ...also just quickly referencing in for the airspeed for our rotate. 
Pilot 06:42 M: ...Just checking that generally set the right height, above the altitude tape there, checking the cyan figure ....all of the reference number in a different colour, that they are the same, any reference figure is all in cyan. So if you see a blue reference number anywhere, that's it. That's a reference number.'

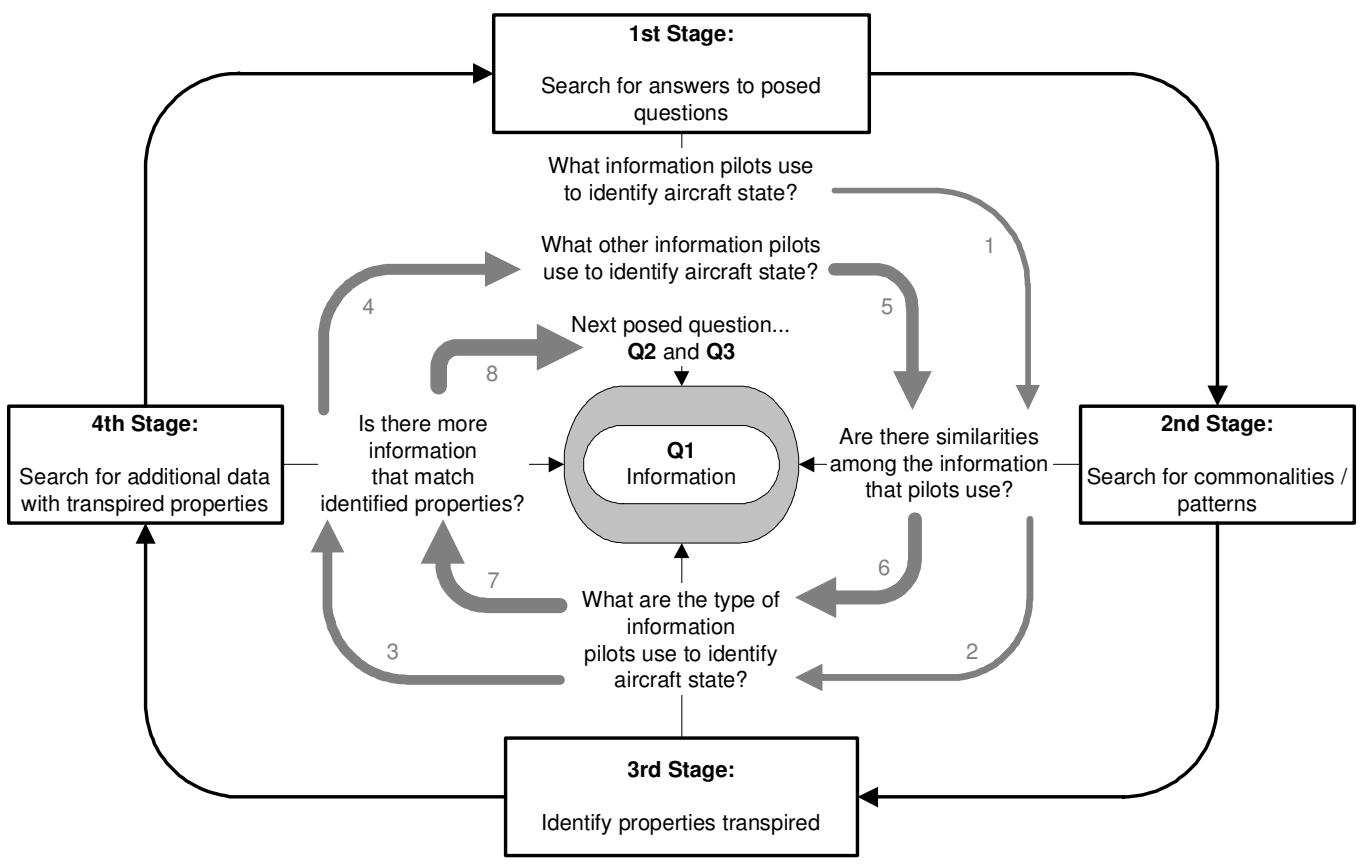

Figure 1 - Evolution of the search

The initial analysis of the above transcript suggests that the pilot is 'referencing' specific information, such as the 'airspeed' to indicate to the pilot the next action 'for our rotate'. From the transcript above we can see that the pilot is 'referencing' several instruments either to verify current aircraft behaviour or using the 'referenced information' to identify the time to active next behaviour, such as in the example 04:42.

This leads to the second stage of the analysis (see Fig. 1) in which patterns and commonalities in the data are identified. A pattern in timing begins to emerge, for example every two minutes throughout the flight the pilot is 'referencing' instruments to establish the aircraft's behaviour. The pilot identifies pieces of information on individual instruments as 'references' to determine the aircraft's behaviour. This is a commonality among the 'referenced information', which we found in the transcript around the words 'referenced to' or 'referred to'. This can be defined as a property of 'referenced information'.

The third stage of analysis involves identifying the properties of already established information. Thus far, the transpired properties of the 'referenced information' are: (1) the information is referenced throughout the flight at similar intervals of time. (2) The information is required to verify current aircraft behaviour. (3) The information is used as a reference (e.g. airspeed or height) to identify the moment of activation for the next behaviour.

The fourth stage involves searching for information that matches the described properties during stage three. Running through the data during the second iteration, keeping in mind the properties described above, the words 'constantly', 'watching' and 'monitor' appear to point at information surrounding them that possess listed properties. Hence, we are searching the transcript further to find words, such as the words 'constantly', 'watching' and 'monitor'. Consider the following examples:

Pilot 06:28: ...he is constantly watching, if I haven't busted a height (i.e., pilot jargon for - to break Air Traffic Control altitude restriction), airspeed or a heading or whatever..

Pilot 11:10: ...I'm watching the speed caret come up and go above the wing, because we want to accelerate, but as to how much that goes before you get to 210 knots it's something that I had to constantly monitor, once I got to 210 knots, then I had to pull power back making sure the caret was on the wing. So it did not raise the workload a great deal, but it did a little bit. There is nothing that really tells you after 210 knots at this height that you need to set this power. 
From the analysis of a complete flight it appeared that the timing of a pilot's comments containing words 'referenced to' or 'referred to, 'constantly', 'watching' and 'monitor', fell into two-minute cycles. The property of all information surrounding these words are also relevant to aircraft behaviour, both verifying current aircraft behaviour (see transcript 06:28) and identifying the moment of activation for the next behaviour (see transcript 11:10). The data is then searched again using the $4^{\text {th }}$ stage of analysis for the words that are similar in meaning to 'referenced to', 'constantly', 'watching and 'monitor'. Doing this, a new fourth property is seen to transpire.

After examining what the data uncovered during the $4^{\text {th }}$ stage, the new property of 'referenced information' established itself. The 'referenced information' was compared to some other features to establish its correct or required position. This can be observed in the following two comments by the pilots, 'I' $m$ watching the speed caret come up and go above the wing' and 'then I had to pull power back make sure the caret was on the wing'. The 'referenced information' here is the speed caret symbol and it is compared to a stationary relatively unchanging reference, the wing symbol on the display.

In both instances the 'referenced information' (i.e., the caret) would have no significance if it was not referenced against another feature (i.e., the wing) that was constant and unchanging relative to a monitored symbol. Thus, a new property of the 'referenced information' is established, i.e., the reference should be constant, unchanging and relative to another feature. Then again, to make sure it is not only specific to this piece of data, a next iteration through already collected data is required. All data has to be analysed again keeping in mind all four established properties.

Having analysed all captured video footage and transcripts from eight flights (i.e., four automated and four non-automated) is was discovered that the information (i.e., according to the first posed question Q1) pilots used to identify the aircraft behaviour and to establish the point in time to activate the next event in both flights, automated and non-automated, was the same.

The properties of this type of 'referenced information' are:

- It is referenced throughout the flight at similar time intervals (e.g. two-minute cycles)

- It is required to verify current aircraft behavior

- It is required to maintain aircraft behavior

- It identifies specific conditions, limitation or boundaries of the system

- It is used to identify the moment of activation of next event/behavior/maneuver

- It is usually connected to other feature/or relative to them

- It is compared to other constant and unchanging features on the display

- When it crosses another feature it becomes a complete symbol (e.g. the wing and caret)

- Pilots have a picture in mind of how this 'referenced information' should align and wait for that moment of alignment to signify the next event

Q2 - Does the information have structure and if so, what is that structure?

The second question is now placed at the centre of Figure 1 entitled 'Q2 - Structure'. The same iteration through four stages has been undertaken. Initial iterations through data showed that pilots in fact had and used 'information structures' that helped them assemble and recall information. These 'information structures' became apparent when pilots used similar types of information in the same order. The scrupulous reading of the transcripts and the reviewing the captured video footage, produced the following information structures:

- $\quad$ Air Traffic Control call

- $\quad$ ATIS (i.e., Automatic Terminal Information Service) announcement

- Structure of a navigation plate (see table 3)

- Brief (e.g., Take-off, Landing) also has a structure

- Operating procedures

- Checklists

The 'structure of information' was found to be either imposed by something physical in the cockpit, such as a display layout, a navigation plate, or it was imposed by an operating procedure. The table 3 below shows two identified 'information structures' (i.e., Take-off brief and Navigation Brief). The real-time data column contains the original transcript from the flight and the 'cued-recall-debrief interview' column provides pilots comment on memorising information in a specific order provided on the plate. This 'information structure' is also reinforced by the operating procedure, which specifies the order in which the information is read from the navigation plate. 


\begin{tabular}{|c|c|c|c|}
\hline $\begin{array}{l}\text { TIME } \\
\text { LINE }\end{array}$ & $\begin{array}{l}\text { SEQUIENCE } \\
\text { OF EVENTS: }\end{array}$ & $\begin{array}{c}\text { STEP TWO: } \\
\text { REAL-TIME DATA }\end{array}$ & $\begin{array}{c}\text { STEP ONE: } \\
\text { CUED-RECALL-DEBRIEF INTERVIEW }\end{array}$ \\
\hline 03:08 & $\begin{array}{l}\text { TAKE-OFF } \\
\text { briefing }\end{array}$ & $\begin{array}{l}\text { 'Glenfield } 1 \text { departure out } \\
\text { of here runway } 10 \text {; plate } \\
\text { stated } 4 \text { October } 2001 \text {, no } \\
\text { amendments; gradient } \\
\text { required } 3.3 \% \text {, which we } \\
\text { can do; track } 095 \text { and } \\
1 \text { TAC or } 1000 \text { feet, } \\
\text { which ever is later, turn } \\
\text { right, track } 170 \text { to } \\
\text { intercept } 144 \text { for } \\
\text { Richmond NDB, track to } \\
\text { Glenfield then as } \\
\text { cleared.' 'Copy' }\end{array}$ & $\begin{array}{l}\text { 'All that is just interpreting what's on the plate there } \& \\
\text { by briefing it, it's actually putting into, right in to our } \\
\text { minds, instead of always refer to it, some of it can be } \\
\text { done from memory. And usually what I will do with } \\
\text { departure, some of the departures would be quite long } \\
\text { and complex. However, you really cannot keep all of } \\
\text { that information in your head, so what you do is brief } \\
\text { the First (i.e., First Officer - the co-pilot) or you just } \\
\text { remember two to three instructions, so like maintain } \\
\text { heading } 095,1000 \text { feet or } 1 \text { TAC. Next what I'm going } \\
\text { to do is turn, right turn on TACAN distance. TACAN is } \\
\text {... a type of DME (i.e., Distance Measuring Equipment). }\end{array}$ \\
\hline
\end{tabular}

Table 3 - Transcript of two steps of the approach

Structuring information appears to be helpful to pilots in recalling and executing actions. Structuring of information happens during 'briefings', such as the brief before the flight or take-off. Structuring information helps pilots to remember 'information' at crucial point during the flight. Here is an example:

Pilot 17:55: I am also, next thing I'm looking at validating the ILS by that outermarker check height again. And PNF (i.e. Pilot-Not-Flying) briefed a little bit before, as to what the height (i.e. 1295 feet), distance (i.e. 4.7 miles) we were looking for, so that's a next step.

This transcript shows the 'information structure' that is purposely placed along the timeline of the flight. The events of the flight are announced in the order that they were briefed earlier.

We analysed data from all flights and having iterated through all four stages of the analysis, we again established that the 'information structures' pilots used where not different between those used in automated and in non-automated flights.

Q3 - Do pilots have strategies in assembling and using information to identify aircraft state?

Q3 is the last main posed question that focuses on identifying 'information strategies' that pilots use to help them deal with vast amount of information. As from the previous example it can be seen that pilots utilise existing information structures to recall and assemble required information. It appears that pilots use this 'information strategy' throughout the flight to assemble 'referenced information' (i.e., the information identified in Q1 section).

Another obvious strategy pilots used was a 'scan'. This 'information strategy' was used to collect and update information they already knew about the state of the aircraft. We searched to this word 'scan' in the transcript to identify how often and for what type of information pilots use this strategy. The word 'scan' appears over ten times in just a single flight transcript. See one example below:

Pilot 04:53: All I'm looking for there on the PFD (i.e., Primary Flight Display), now my focus has come in inside once we are far away from the ground. All I am doing is getting my attitude and heading set on the PFD, so I'm concentrating on putting the climb-dive marker where I want it. Obviously we don't have any reference information there now, so I am just looking at the reference, the pitch ladder. So that's all. How many degrees I want \& I was looking for about 7 degrees nose up there. That's usually a good figure to remember. As accelerating at a nice rate, but not to quick, so you are not going to over speed the gear or anything like that. The other part of my scan is looking down at the compass card and quickly referencing and having a look at the level on there as to what heading I am flying.

Pilots also use 'a scan strategy' to maintain and to verify the aircraft's behaviour. Pilots identify, assemble and position 'referenced information' in their mind at similar time intervals along a flight timeline prior the flight or during a flight brief. The aligned 'referenced information' is then checked during the flight at assigned time intervals for accuracy against initial identified 'referenced information'.

Pilots also employ 'a brief strategy' to construct the information before the entire flight during a preflight briefing session and before each significant part of the flight throughout the flight. Pilots are constructing and aligning 'referenced information' in their mind, which can either be a visual cue in the environment (e.g. a view of the airport approaching from South) or on the display (e.g. altitude 
reading). Pilots would either image or draw the flight on the board before the flight, establishing important 'referenced information' (e.g. Navigation point on the display or Altitude). In the example 03:08 (table 3) the pilot uses “...track 015 (i.e., heading) and 1TAC (i.e., navigation point) or 1000 feet (i.e., altitude)" as major information 'references' to help establish significant points in flight that would indicate the time for an action to be executed "...turn right, track 170". The pilot's comments state that, '... by briefing it (i.e., take-off), it's actually putting into, right in to our minds...some of it can be done from memory'.

These 'information strategies' were identified through iteration of four stages of analysis, refining the key words or structure of sentences that were repeated several types. For example, comment 03:08 shows how pilots briefed the departure and several minutes later the co-pilot executed briefed actions simultaneously recalling information briefed (e.g. 'heading 095, 1000 feet or 1 TAC'). To identify similar strategies we searched the transcript for similar situations where information briefed was recalled.

We also found that pilots used an 'information strategy' to help them recall required information by constructing future references using a timeline-sequence structure, which later in the flight triggered recollection of the required action to be executed.

These are only few examples of the strategies employed by the pilots in information utilisation identified in automated and non-automated flight.

\section{Information Evolution Throughout the Flight}

The four-stage analysis resembles tracing a 'spider-web' of information and no matter where the researcher starts until most of the routes that makes the picture complete are identified the analysis is not finished.

The analysis revealed that all information pilots use is connected or related to other pieces of information via an 'information structure' or an 'information strategy'. The information pilots used was constantly evolving. Pilots used strategies to update and generate new references (i.e., 'referenced information') to keep up with evolving information. Pilots applied 'information strategies' using 'referenced information' and 'information structures' to maintain flight path and the required aircraft behaviour.

As a result of the analysis a model emerged illustrating how pilots acquire and use information. A model consisting of eight phases is given that shows the spiral of how pilots acquire and use information, how information evolves and how pieces of information relate to other 'referenced information' (see Fig. 2). The model evolves into a spiral at each progression through eight phases, representing pilot's progression of acquiring information, gain knowledge and experience.

The first phase of information progression represents that the pilot has an existing knowledge based on experience, for example an existing 'information structure', such stages of the flight or steps in the operating procedure. For example, Air Traffic Control calls, and flight briefs, are strategies that the pilot has acquired through training and on-line operation. This first phase would also include a request for new flight information. At this point the pilots will build on the existing knowledge (i.e., previously used 'referenced information' and 'information structures') and experience (e.g. 'information strategies', such as 'brief'), adding new information to old 'information structures' for example.

The second phase represents the acquisition of all new information related to the flight. All information regarding this flight will be introduced during this phase, the brief, the planning of the route, route related weather and restrictions. The second phase is also the beginning of the information acquisition phase and the processing of new information that continues through the next four phases.

The third phase of information acquisition involves identifying alternative airports and all the related information for alternative arrangements, such as additional route calculations, relevant weather and restrictions on route (e.g. height, speed, no-fly zone). New flight regulations and restrictions that are relevant or have been introduced will also be introduced in this phase.

The fourth phase is the choice of information acquisition and this is where new solutions, identification, division of work and problems are assigned between pilots. At this phase new information is generated out of all information previously acquired prior to the flight. The calculation of relevant-to-the-flight-information-references happens at this phase. If this phase is to be associated with a flight stage, it would be a 'brief before take-off', a 'brief' before a significant event or a 'brief' due to a change in original flight plan, containing more new information. 


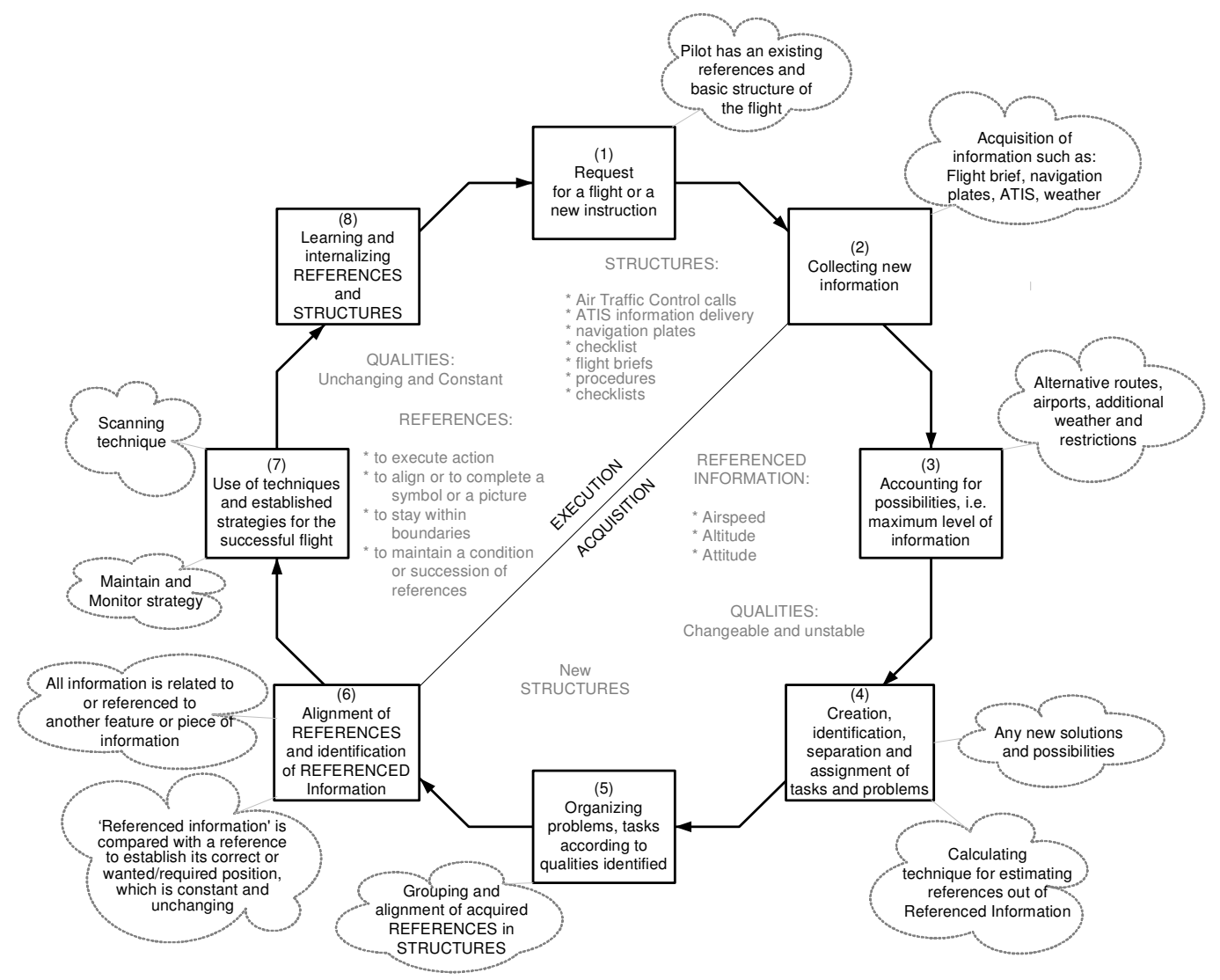

Figure 2. Evolution of Information Flow

The fifth phase involves organising information. This is where pilots group and align references that they later use in flight. At this phase the information gets sorted into structures to assist pilots in implementation of their strategies.

The sixth phase is the end of information acquisition and beginning of information use. All new acquired 'referenced information' and 'information structures' are compared with existing references and structures that pilots hold in their mind. All pieces of information fall into place; the blend of information, structures, new and old happens in this phase in the pilots mind. Pilots compose the references in their mind and position them on the display relative to other information or relative to already existing references. All information is connected and dependent on each other, and the links are established. This is the point of clarity. In this phase the information is not likely to change its position, unless a change in flight plan or situation occurs.

The seventh phase is the flight execution phase. All the 'information strategies', for example, 'maintain', 'monitor' and 'scan', pilots use to fly the aircraft are implemented here on the basis of newly acquired and organised information, and previous experience.

The eighth phase involves turning all newly attained information, such as 'referenced information' and 'information structures' into knowledge and experience. The iterations through the spiral bring the pilot to a new level, the phase one, with added knowledge and experience from the last flight. New iterations through the eight phases is triggered by a new flight or a change to the flight plan.

\section{Conclusion}

This paper demonstrated how a three-step approach without interruptions can elicit information that pilot require to fly the aircraft efficiently. The first two steps involved the use a headmounted videocamera's that provided a pilot with his own point-of-view to be a valuable cue to recollect the activities in flight and guide the researcher to information that is vital in a complex and demanding environment. Instead, of a researcher imposing their interpretation of the information structure, the relevance and meaningfulness of the information structure is derived from pilots' activities. That is, in our study throughout the flight the pilot's own-point-of-view, their activities and pilots' recollection of events were the source of all data acquired. 
As a result of this study a model of Information Flow (Fig. 2) emerged, that shows how 'referenced information', 'information structures' and 'information strategies' evolve. This has been depicted diagrammatically (see Fig. 2) and shows how complex the evolution of information is during piloting of the aircraft. It shows how information is coming from many sources, is constantly changing, and being affected by events throughout the flight. Additionally, the model shows that pilot's have stored 'referenced information', 'information structures' and 'information strategies', which are regularly used and evolve. In related work (Solodilova, Lintern \& Johnson, 2005) we show that these references, structures and strategies are poorly supported in current displays and consequently can be a source for pilots' confusion and misunderstanding of automation. In a previous paper (Solodilova \& Johnson, 2004) we show that the use of references, structures and strategies to inform design can produce more efficient displays, where pilots perform twice as fast and with less error.

A further conclusion from this data analysis is that pilots already have existing information structures and pieces of information that are significant to them. We need to use the results of this study indicating how pilots use the information, structures and strategies to the advantage of pilots and to help design engineers in the design of information space in the glass cockpit.

The information layout of new glass cockpit interfaces should support 'referenced information', 'information structures', and 'information strategies' that evolved for over a century. The substantial amount of references and structures that pilots use, learned since they started to fly, should be the main source inspiration. Instead of inventing new ways of presenting information Pilot's own information use strategies should be supported and used.

\section{Acknowledgments}

This work is being funded by the EPSRC (grant number GR/R40739/01) and supported by QinetiQ and Westland Helicopters. We would like to thank the pilots, who participated in the flight observations study and in particular Squadron Leader Mark Thoresen of 85WG RAAF Base Richmond, NSW, Australia, for organizing and making this research possible.

\section{References}

Cantor, D., Andreassen, C. \& Waters, H. (1985). Organization in visual episodic memory: Relationships between verbalised knowledge, strategy use, and performance, Journal of Experimental Psychology, 40:218-232.

Demagalski, J., Harris, D., Salmon, P., Stanton, N. A., Young, M. S., Marshall, A., Waldman, T., \& Dekker, S. W. A. (2002). Design induced errors on the modern flight deck during approach and landing. In: Proceedings of HCI-Aero 2002, MIT, Cambridge, MA, pp.173-178.

Heymann, M. \& A. Degani (2002). Constructing Human-Automation Interfaces: A Formal Approach. In: Proceedings of the International Conference on Human Computer Interaction in Aeronautics, Cambridge, Massachusetts, AAAI Press, California, USA, pp.119-124

McLennan, J., M. Omodei, M. \& Rich, D. (1997). Using a head-mounting video camera system and two-stage debriefing procedure: A new approach to advances skills training in industry. Swinburne University of Technology Institute of Social Research, Melbourne, Australia.

McLennan, J., M. Omodei, M., \& Wearing, A. (2000). Cognitive Processes of First-on-Scene Fire Officers in Command at Emergency Incidents as an Analogue of Small-Unit Command in Peace Support Operations. 2nd International Workshop on The Human in Command: Peace Support Operations, Breda, The Netherlands.

Omodei, M., Wearing, A., \& McLennan, J. (1998). Integrating Field Observation and Computer Simulation to Analyse Command Behaviour. In: Proceedings of the NATO RTO Workshop on The Human in Command, June 1998, Kingston, Canada.

Neisser, U. (1976). Cognition and Reality: Principles and Implication of the Cognitive Psychology, Freeman, San Francisco, USA.

Newman, R. L. \& Greeley, K. W. (2001). Cockpit Displays: Test and Evaluation. Ashgate, Aldershot.

Omodei, M., Wearing, A., \& McLennan, J. (1997). Head-mounted video recording: A methodology for studying naturalistic decision making. In R. Flin, E. Salas, M. Strub and L. Martin (Eds.) Decision Making Under Stress: Emerging Themes and Applications. (pp. 137-146) Ashgate, Aldershot.

Sarter, N. B. \& Woods, D. D. (1994). Pilot Interaction with cockpit Automation II: An Experimental Study of Pilots' Model and Awareness of the Flight Management System. The International Journal of Aviation Psychology, 4 (1):1-28.

Sarter, N. B. \& D. D. Woods (1995). How in the world did we get into that mode? Mode error and awareness in supervisory control. Human Factors, 37 (1):5-19. 
Solodilova, I., Lintern G., \& Johnston, N. (2003) The Modern Commercial Cockpit As a MultiDimensional, Information-Action Workspace. In: Proceedings of the 12th International Symposium on Aviation Psychology, 14-17 April 2003, Dayton, Ohio, USA, pp.1096-1101.

Solodilova, I., \& Johnson, P. (2004) 'Mind References' in the Glass Cockpit: An Experimental Study. In: Proceedings of the HCI Aero 2004, Toulouse, France, [CD-ROM].

Solodilova, I., Lintern G., \& Johnson, N. (2005) A Mind-Reference Framework for design and evaluation of intuitive and natural interfaces. In: Proceedings of the 13th International Symposium on Aviation Psychology, 18-21 April 2005, Oklahoma City, Oklahoma, USA. In press

\section{Apppendix}

\section{From original two-stage to a modified cued-recall-debrief procedure with the use of head-mounted camera on the operator}

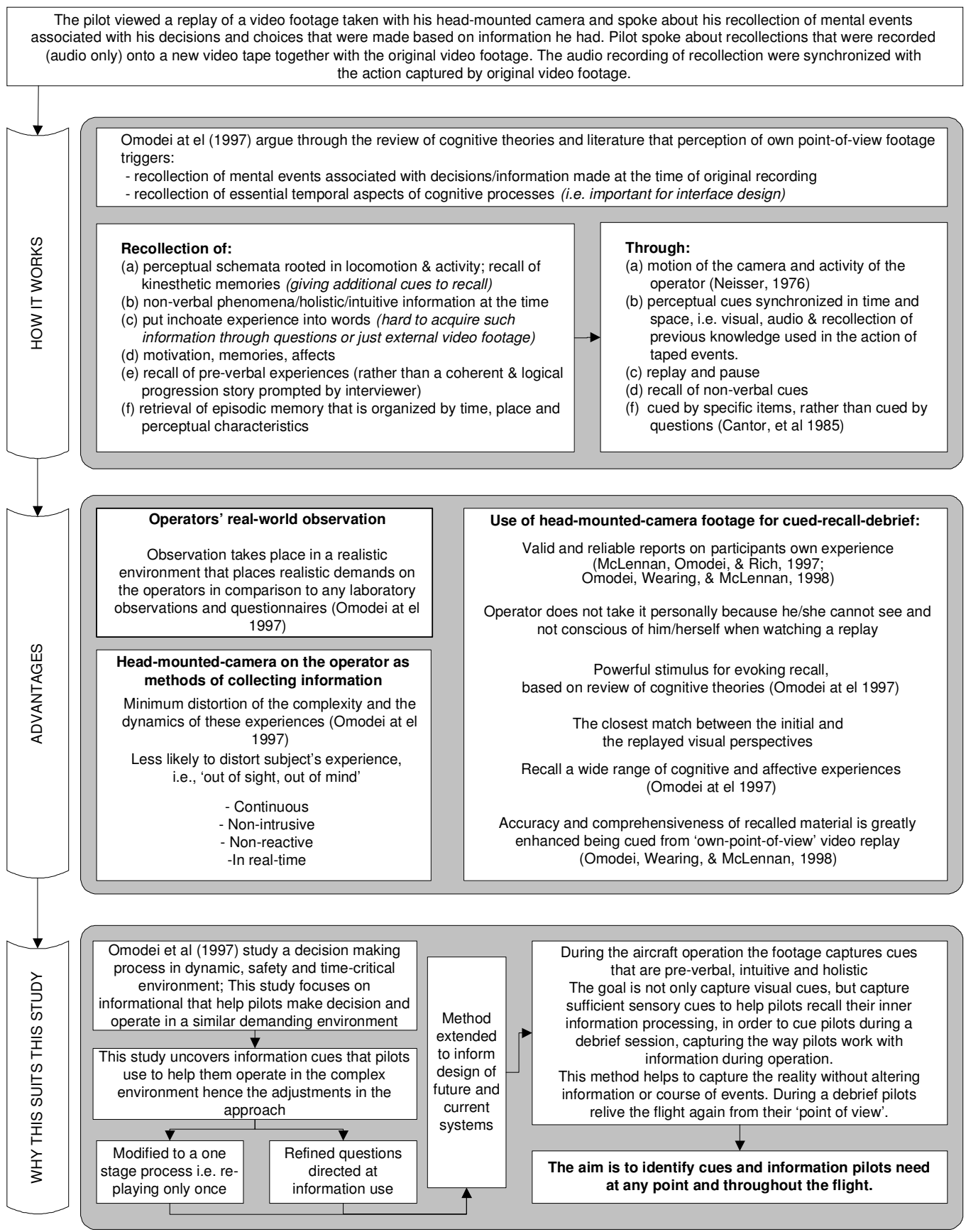

